\title{
SPECTRAL CURVES FOR HYPERGEOMETRIC HURWITZ NUMBERS
}

\author{
JAN AMBJØRN ${ }^{\dagger}$ AND LEONID O. CHEKHOV*
}

\begin{abstract}
We consider multi-matrix models that are generating functions for the numbers of branched covers of the complex projective line ramified over $n$ fixed points $z_{i}, i=1, \ldots, n$, (generalized Grotendieck's dessins d'enfants) of fixed genus, degree, and the ramification profiles at two points, $z_{1}$ and $z_{n}$. Ramifications at other $n-2$ points enter the sum with the length of the profile at $z_{2}$ and with the total length of profiles at the remaining $n-3$ points. We find the spectral curve of the model for $n=5$ using the loop equation technique for the above generating function represented as a chain of Hermitian matrices with a nearest-neighbor interaction of the type $\operatorname{tr} M_{i} M_{i+1}^{-1}$. The obtained spectral curve is algebraic and provides all necessary ingredients for the topological recursion procedure producing all-genus terms of the asymptotic expansion of our model in $1 / N^{2}$. We discuss braid-group symmetries of our model and perspectives of the proposed method.
\end{abstract}

\section{INTRODUCTION}

Hurwitz numbers enumerate combinatorial classes of ramified mappings $f: \mathbb{C} P^{1} \rightarrow \Sigma_{g}$ of the complex projective line onto a Riemann surface of genus $g$. The terms single or double Hurwitz numbers describe the cases in which ramification profiles (defined by the corresponding Young tableauxes $\lambda$ or $\lambda$ and $\mu$ ) are respectively fixed at one $(\infty)$ or two ( $\infty$ and 1$)$ distinct points whereas we assume the existence of $m$ other distinct ramification points with only simple ramifications.

Generating functions for Hurwitz numbers possess a number of remarkable properties: integrability of character expansions was observed in [31] and further developed in [5]. A. Yu. Orlov and Shcherbin [39], [40] and Okounkov [37] and Pandharipande [38] identified the exponential of the generating function for double Hurwitz numbers with a tau-function of the KadomtsevPetviashvili (KP) hierarchy. Note that the class of models enjoying the KP hierarchy properties happens to be rather wide. General conditions on KP tau functions were first formulated by Takasaki [41] and later reformulated by Goulden and Jackson [24] using the Plucker relations.

In [7], [8], we considered Hurwitz numbers corresponding to another ramification pattern in which we have a fixed number $n$ of ramification points with arbitrarily complex ramification profiles at every point. The case $n=3$ treated in [7] is in one-to-one correspondence with the celebrated Grothendieck's dessins d'enfants. This case was first considered by Orlov and Shcherbin [39] who demonstrated that the exponential of the corresponding generating function is a tau function of the KP hierarchy. Moreover, the authors of [39] proposed a description of a wider class of generating functions for hypergeometric Hurwitz numbers with $n$ of ramification points in $\mathbb{C} P^{1}$ : we fix profiles at two of these points, taking the sum over profiles at all other points with weights proportional to the lengths of the remaining $n-2$ profiles. Harnad and Orlov [28] demonstrated that all these generating functions are in turn tau functions of the KP hierarchy.

${ }^{\dagger}$ Niels Bohr Institute, Copenhagen University, Denmark, and IMAPP, Radboud University, Nijmengen, The Netherlands. Email: ambjorn@nbi.dk.

*Steklov Mathematical Institute and Interdisciplinary Scientific Center J.-V. Poncelet, Moscow, Russia; Michigan State University, East Lansing, USA. Email: chekhov@mi.ras.ru. 
The relation of branched coverings of the complex plane to matrix models was established almost immediately upon the progress in both these branches of combinatorics in the early 90 th, and the first proposal of a matrix-model description of Belyi morphisms was presented by Di Francesco and Itzykson [18]. Their model, inspired by the Kontsevich matrix model, described the two-profile Hurwitz numbers for clean Belyi morphisms (with only square-root branchings at the third point). We know now that this model falls outside of the KP hierarchy class and no reliable method for constructing a large- $N$ asymptotic expansion of this model has been found since its formulation. On the other hand, matrix-model descriptions of single Hurwitz numbers were successfully developed in a series of papers by de Mello Koch and Ramgoolam [19], [20] in cases where we restrict the possible orders of branchings; we then obtain the simplest Hermitian one-matrix model with a polynomial potential of degree that is the maximum allowed order of branching.

The interest in Hurwitz numbers corresponding to Belyi pairs was revived in the mathematical community by Zograf [42] (see also [30]) who provided recursion relations based on a cut-and-joint procedure for the generating function of Grothendieck's dessins d'enfants.

In [7], we develped a matrix-model description of Belyi morphisms, clean Belyi morphisms, and two-profile Belyi morphisms: the three corresponding matrix models are the standard Hermitian one-matrix model with a logarithmic addition to the potential, the Kontsevich-Penner matrix model [16, and the generalized Kontsevich model [32, 33] also with a logarithmic term (it is the BGW model of [35]). The multi-matrix-model representation for the hypergeometric Hurwitz numbers then followed [6] in the form of a matrix chain, albeit with a complicated interaction between matrices in the chain. We have proposed another chain-matrix-model description of hypergeometric Hurwitz numbers in [8], also with a nonstandard interaction between the matrices in the chain, for which we were able to construct the spectral curve (for the case $n=4)$. In our approach, we fix profiles at two ramification points, fix the length of the profile at the third point, and fix the total length of profiles at other $n-3$ points. In this case, we can derive the spectral curve equation in the framework of the $1 / N^{2}$-expansion. Armed with this spectral curve and two meromorphic differentials $d x$ and $\omega_{1}(x) d x$ on it (the second differential comes from the resolvent of the first matrix in the matrix chain) we can apply the topological recursion procedure for constructing consecutively the whole series of the $1 / N^{2}$-expansion, thus obtaining hypergeometric Hurwitz numbers for complex curves of arbitrary genus $g$ (the corresponding free-energy term is proportional to $N^{2-2 g}$ ).

It turned out that the chain multi-matrix model with the same interaction $\operatorname{tr} M_{i} M_{i+1}^{-1}$ between neighbour matrices in the chain was proposed by G. Akemann and collaborators [1], 2] in the context of problems of joint probability distribution in quantum informatics. They constructed kernels for such models in terms of Meijer G-functions, which we also briefly mention at the end of Sec. 2, but did not address problems of constructing spectral curves.

We first recall the famous relation between Belyi pairs and Galois groups.

Theorem 1.1. (Belyi, [11]) A smooth complex algebraic curve $C$ is defined over the field of algebraic numbers $\overline{\mathbb{Q}}$ if and only if we have a nonconstant meromorphic function $f$ defined on $C\left(f: C \rightarrow \mathbb{C} P^{1}\right)$ that is ramified only over the points $0,1, \infty \in \mathbb{C} P^{1}$.

A Belyi pair $(C, f)$ is the curve endowed with such a function. Let $g$ be the genus of $C$ and $d$ the degree of $f$. If we take the preimage $f^{-1}([0,1]) \subset C$ of the real line segment $[0,1] \in \mathbb{C} P^{1}$ we obtain a connected bipartite fat graph with $d$ edges. Vertices of this graph are preimages of 0 and 1 and the cyclic ordering of edges entering a vertex is fixed by orientation of the curve $C$. Grothendieck had then formulated the following lemma.

Lemma 1.2. (Grothendieck, [25]) There is a one-to-one correspondence between the isomorphism classes of Belyi pairs and connected bipartite fat graphs. 


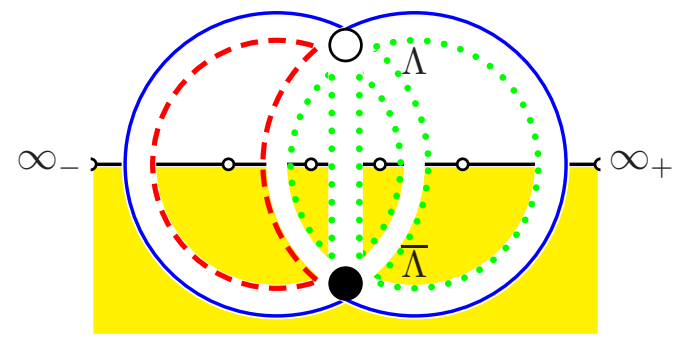

FiguRE 1. The generalized Belyi fat graph $\Gamma_{1}$ corresponding to the case of $n=5$ ramification points $(\infty,-(1+\sqrt{5}) / 2,0,1$, and $(3+\sqrt{5}) / 2$ denoted by small white circles) of Sec. 3. The generalized Belyi pair $\left(\mathbb{C} P^{1}\right.$, id $)$ corresponds to this graph; $\infty_{ \pm}$ indicate directions of approaching the infinite point in $\mathbb{C} P^{1}$. The symbols $\Lambda$ and $\bar{\Lambda}$ indicate external field insertions in the matrix-model formalism of Sec. 2. For example, this graph contributes the term $N^{2} \gamma_{1} \gamma_{2} \gamma_{3}^{2} t_{1} \operatorname{tr}(\Lambda \bar{\Lambda})$.

A Grothendieck dessin d'enfant is correspondingly a connected bipartite fat graph representing a Belyi pair. It is well known that we can naturally extend the $\operatorname{dessin} f^{-1}([0,1]) \subset C$ corresponding to a Belyi pair $(C, f)$ to a bipartite triangulation of the curve $C$.

The hypergeometric Hurwitz numbers correspond to generalized Belyi pairs, which are mappings $\left(f: C \rightarrow \mathbb{C} P^{1}\right)$ with allowed ramifications over $n$ fixed points $z_{i} \in \mathbb{C} P^{1}, i=1, \ldots, n$. We then have the splitting of the curve $C$ into bipartite $n$-gons with edges of $n$ colors (the corresponding fat graphs are then coverings of the basic graph depicted in Fig. 1 for $n=5$, which is the main example under consideration in this paper). We assign color to an edge depending on which of $n$ segments of $\mathbb{R} P^{1-f^{-1}}\left(\left[\infty_{-}, z_{2}\right]\right) \subset C, f^{-1}\left(\left[z_{2}, z_{3}\right]\right) \subset C, \ldots, f^{-1}\left(\left[z_{n-1}, z_{n}\right]\right) \subset C$, $f^{-1}\left(\left[z_{n}, \infty_{+}\right]\right) \subset C$-its image intersects (we identify $z_{1}$ with the infinity point and let $\infty_{ \pm}$ indicate the directions of approaching this point along the real axis in $\left.\mathbb{C} P^{1}\right)$. Each face of the dual partition then contains a preimage of exactly one of the points $z_{1}, \ldots, z_{n}$, so these faces are of $n$ sorts (bordered by solid, dotted, or dashed lines in the figure). We call such a graph a generalized Belyi fat graph.

The type of ramification at infinity is fixed by the (unordered) set of solid-line-bounded faces of a generalized Belyi fat graph: the order of branching is $r$ for a $2 r$-gon, so we introduce the generating function that distinguishes between different types of branching at infinity, or at $z_{1}$. Moreover, we also distinguish between different types of ramifications at the $n$th point (the point $(3+\sqrt{5}) / 2$ in Fig. 1). We are going therefore to produce a two-profile generating function for Hurwitz numbers; branching patterns at two distinct points can be represented by the corresponding Young tableaux. We let $k_{i}$ denote the numbers of respective cycles (preimages of the points $z_{i}$ on the Riemann surface $C$ ) and let $k_{1}^{(r)}$ and $k_{n}^{(r)}$ denote the numbers of cycles of length $2 r$ centered at pre-images of the respective points $z_{1}$ and $z_{n}$ in a generalized Belyi fat graph. An example of a 19 -fold covering of $\mathbb{C} P^{1}$ by a torus in the case $n=4$ is depicted in Fig. 2

As was shown in [6] and [28], the exponential of the generating function

$$
\mathcal{F}\left[\left\{t_{m}\right\},\left\{\mathfrak{t}_{r}\right\}, \gamma_{2}, \ldots, \gamma_{n-1} ; N\right]=\sum_{\Gamma} \frac{1}{|\operatorname{Aut} \Gamma|} N^{2-2 g} \prod_{r=1}^{\infty} t_{r}^{k_{1}^{(r)}} \prod_{s=1}^{\infty} \mathfrak{t}_{s}^{k_{n}^{(s)}} \prod_{j=2}^{n-1} \gamma_{j}^{k_{j}}
$$

is a tau function of the KP hierarchy either in times $t$ or $\mathfrak{t}$. A matrix-model description of this generating function was proposed in the above papers, and nonlinear cut-and-join equations were constricted in [6], but the possibility of solving the model in topological recursion terms (see [22], [14], [15]) remained obscure. A matrix model describing a subclass of generating functions (1.1) with $\gamma_{3}=\gamma_{4}=\cdots=\gamma_{n-1}$ and with arbitrary $\gamma_{2}>\gamma_{3}$ was proposed in [8]. 


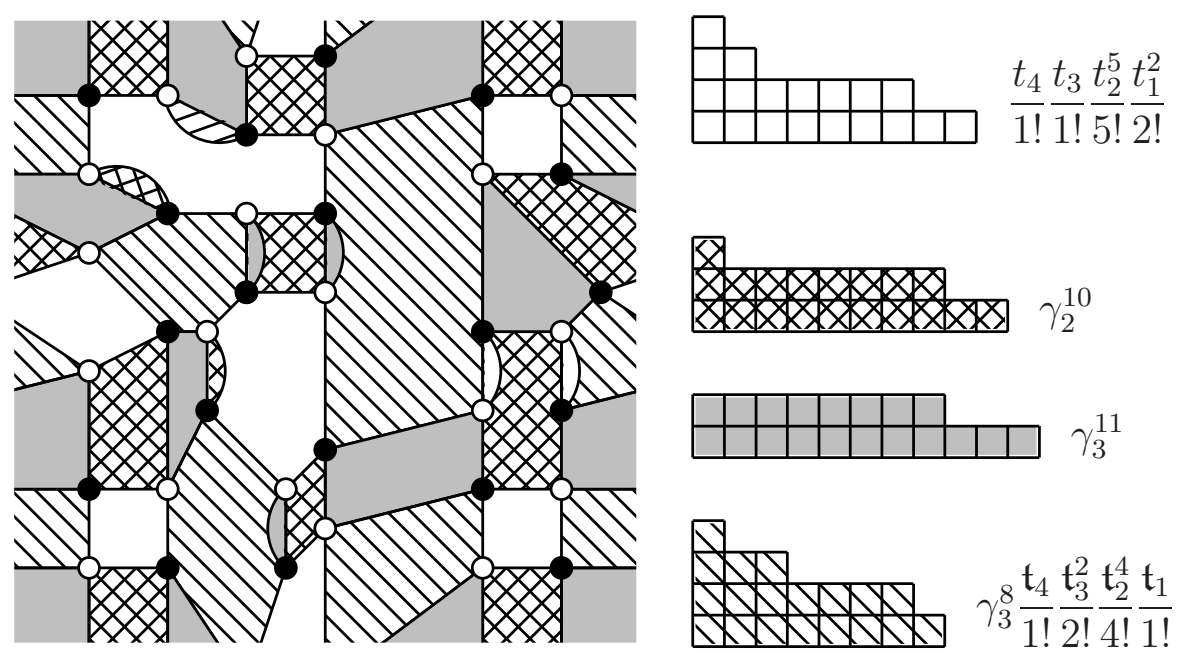

Figure 2. An example of 19-fold covering of $\mathbb{C} P^{1}$ by a torus; we identify the upper and lower as well as left and right boundaries of the square; in the left side we present the corresponding Young tableauxes for collections of polygons of all four colors indicating the weights with which these tableauxes enter the generating function. All separating lines in the right figure are assumed to be double lines.

An advantage of this model is that it can be solved within a topological recursion method adapted to chain of matrices with nonstandard interaction terms. In [8], only the case of one intermediate matrix $(n=4)$ was presented; in the present paper, we extend it to the case $n=5$; the structure of solution for a general $n$ will then become clear.

We thus solve a matrix model whose free energy is the generating function

$$
\mathcal{F}\left[\left\{t_{m}\right\},\left\{\mathfrak{t}_{r}\right\}, \gamma_{2}, \gamma_{3} ; N\right]=\sum_{\Gamma} \frac{1}{|\operatorname{Aut} \Gamma|} N^{2-2 g} \prod_{r=1}^{\infty} t_{r}^{k_{1}^{(r)}} \prod_{s=1}^{\infty} \mathfrak{t}_{s}^{k_{n}^{(s)}} \gamma_{2}^{k_{2}} \gamma_{3}^{k_{3}+\cdots+k_{n-1}},
$$

where $N, \gamma_{2}, \gamma_{3}, t_{r}$, and $\mathfrak{t}_{r}$ are formal independent parameters and the sum ranges over all (connected) generalized Belyi fat graphs. Our matrix model contains an external matrix field $\Lambda=\operatorname{diag}\left(\lambda_{1}, \ldots, \lambda_{\gamma_{3} N}\right)$ producing the corresponding times

$$
\mathfrak{t}_{r}=\operatorname{tr}\left[(\Lambda \bar{\Lambda})^{r}\right]
$$

A general case with all $\gamma_{j}$ different was treated in [1], 2]; we leave the problem of finding spectral curves for these generalized models for future studies.

The structure of the paper is as follows. In Sec. 2, we show that generating function (1.2) is the free energy of a special multi-matrix model represented as a chain of matrices with somewhat nonstandard interaction terms. We describe the braid-group symmetries of this model. This model can be expressed in terms of an integral over eigenvalues of matrices from the corresponding matrix chain in a form similar to that of the standard generalized Kontsevich model (GKM) 32], 33] giving rise to Meijer G-functions. Our main result is a solution to the loop equations of this model for $n=5$ in Sec. 3 describing the spectral curve and two meromorphic differentials on it, i.e., all ingredients necessary for constructing corrections in all genera using the topological recursion. For this, we modify the technique of Eynard and Prats Ferrer [23] to evaluate spectral curves for chains of matrices. We conclude with a discussion of our results.

Throughout the entire text we disregard all multipliers not depending on external fields and times $t_{r}$; all equalities in the paper must be therefore understood modulo such irrelevant factors. 


\section{THE MATRIX MODEL}

2.1. From Hurwitz numbers to the matrix chain. Our main example in this paper will be hypergeometric Hurwitz numbers for coverings of $\mathbb{C} P^{1}$ ramified over five points $(n=5)$. On the first stage, we take into account the profile at the infinity point for which we contract all solid cycles (centered at pre-images of $\infty$ ) assigning the time $t_{r}$ to every contracted cycle of length $2 r$. The thus contracted solid cycles become new vertices.

The matrix-valued fields $B_{i}, i=2, \ldots, n-1$, are general complex-valued matrices such that $B_{2}$ is a rectangular matrix of the size $\gamma_{2} N \times \gamma_{3} N$ and we always assume that

$$
\gamma_{2}>\gamma_{3}
$$

and all other matrices $B_{3}, \ldots, B_{n-1}$ are square matrices of the size $\gamma_{3} N \times \gamma_{3} N$.

The matrix-model integral whose free energy is the generating function (1.2) reads

$$
\int D B_{2} \cdots D B_{n-1} e^{N \sum_{r=1}^{\infty} \frac{t_{r}}{r} \operatorname{tr}\left[\left(B_{2} \cdots B_{n-1} \Lambda \bar{\Lambda} \bar{B}_{n-1} \cdots \bar{B}_{2}\right)^{r}\right]-\sum_{j=2}^{n-1} N \operatorname{tr}\left(B_{j} \bar{B}_{j}\right)}
$$

Here, every vertex contains, besides $2 r(n-2)$ insertions of matrix fields we integrate over, $r$ insertions of the external diagonal matrix field $\Lambda \bar{\Lambda}$; the factor $1 / r$ takes into account the cyclic symmetry of the $2 r$-cycle.

We next perform the variable changing

$$
\begin{aligned}
& \mathfrak{B}_{2}=B_{2} B_{3} \cdots B_{n-1} \\
& \mathfrak{B}_{3}=B_{3} \cdots B_{n-1} \\
& \vdots \\
& \mathfrak{B}_{n-1}=B_{n-1}
\end{aligned}
$$

and assume that all matrices $\mathfrak{B}_{3}, \ldots, \mathfrak{B}_{n-1}$ are invertible (the matrix $\mathfrak{B}_{2}$ remains rectangular). With accounting for the Jacobian of transformation (2.2), the integral (2.1) becomes

$$
\begin{aligned}
& \int D \mathfrak{B}_{2} \cdots D \mathfrak{B}_{n-1} \exp \left\{-\gamma_{2} N \operatorname{tr} \log \left(\mathfrak{B}_{3} \overline{\mathfrak{B}}_{3}\right)-\sum_{j=4}^{n-1} \gamma_{3} N \operatorname{tr} \log \left(\mathfrak{B}_{j} \overline{\mathfrak{B}}_{j}\right)\right. \\
& +\sum_{r=1}^{\infty} N \frac{t_{r}}{r} \operatorname{tr}\left[\left(\mathfrak{B}_{2}|\Lambda|^{2} \overline{\mathfrak{B}}_{2}\right)^{r}\right]-N \operatorname{tr}\left[\mathfrak{B}_{2} \mathfrak{B}_{3}^{-1} \overline{\mathfrak{B}}_{3}^{-1} \overline{\mathfrak{B}}_{2}\right] \\
& \left.-N \operatorname{tr}\left[\mathfrak{B}_{3} \mathfrak{B}_{4}^{-1} \overline{\mathfrak{B}}_{4}^{-1} \overline{\mathfrak{B}}_{3}\right]-\cdots-N \operatorname{tr}\left[\mathfrak{B}_{n-2} \mathfrak{B}_{n-1}^{-1} \overline{\mathfrak{B}}_{n-1}^{-1} \overline{\mathfrak{B}}_{n-2}\right]-N \operatorname{tr}\left[\mathfrak{B}_{n-1} \overline{\mathfrak{B}}_{n-1}\right]\right\} .
\end{aligned}
$$

This expression justifies restrictions imposed on the matrix sizes: we must be able to invert the matrices $\mathfrak{B}_{j}$ with $j=3, \ldots, n-1$ in order to write the corresponding generating function as a free energy of a Hamiltonian of a chain of Hermitian matrices.

We now recall [9] that we can write an integral over general complex matrices $\mathfrak{B}_{i}$ in terms of positive definite Hermitian matrices $X_{i}$ upon the variable changing

$$
X_{i}:=\overline{\mathfrak{B}}_{i} \mathfrak{B}_{i}, \quad i=2, \ldots, n-1 .
$$

All the matrices $X_{i}(i=2, \ldots, n-1)$ are of the same size $\gamma_{3} N \times \gamma_{3} N$. Changing the integration measure for rectangular complex matrices introduces a simple logarithmic term (the Marchenko-Pastur law [34]), and the resulting integral becomes

$$
\begin{aligned}
& \int D X_{2 \geq 0} \cdots D X_{n-1 \geq 0} \exp \left\{N \sum_{r=1}^{\infty} \frac{t_{r}}{r} \operatorname{tr}\left[\left(X_{2}|\Lambda|^{2}\right)^{r}\right]-N \operatorname{tr}\left(X_{2} X_{3}^{-1}\right)-\cdots-N \operatorname{tr}\left(X_{n-2} X_{n-1}^{-1}\right)\right. \\
& \left.\quad-N \operatorname{tr} X_{n-1}+\left(\gamma_{2}-\gamma_{3}\right) N \operatorname{tr} \log X_{2}-\gamma_{2} N \operatorname{tr} \log X_{3}-\gamma_{3} N \operatorname{tr} \log \left(X_{4} \cdots X_{n-1}\right)\right\} .
\end{aligned}
$$




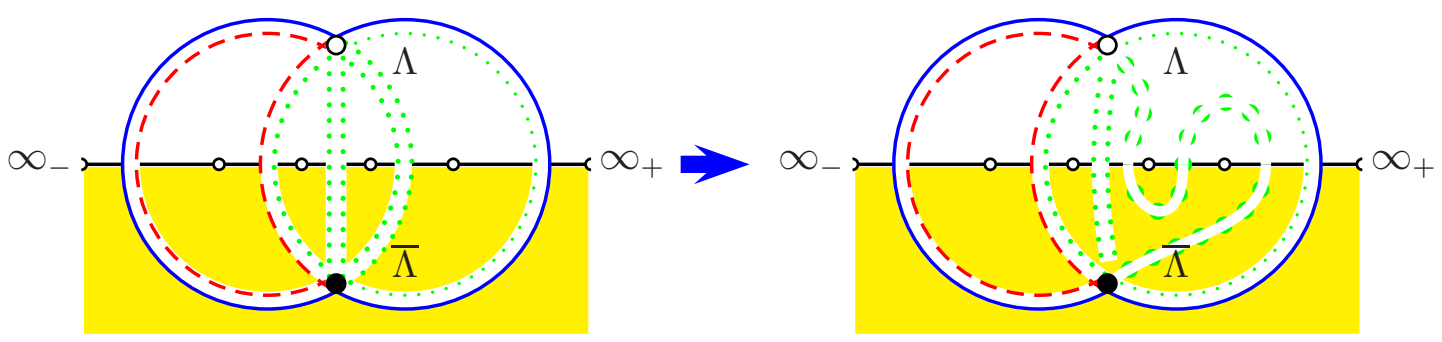

FiguRE 3. The graph transformation resulting in the braid-group transformation permuting the neighbour branching points.

The logarithmic term in $X_{2}$ stabilizes the equilibrium distribution of eigenvalues of this matrix in the domain of positive real numbers; in the case where $\gamma_{2}=\gamma_{3}$, we lose this term and must use the technique of matrix models with hard walls (for a review, see [12]).

Performing a scaling $X_{i} \rightarrow X_{i}|\Lambda|^{-2}$ for all the integration variables, we bring (2.5) to a more familiar form of an integral over a chain of matrices,

$$
\begin{aligned}
& \int D X_{2 \geq 0} \cdots D X_{n-1 \geq 0} \exp \left\{N \sum_{r=1}^{\infty} \frac{t_{r}}{r} \operatorname{tr}\left(X_{2}^{r}\right)-N \operatorname{tr}\left(X_{2} X_{3}^{-1}\right)-\cdots-N \operatorname{tr}\left(X_{n-2} X_{n-1}^{-1}\right)\right. \\
& \left.-N \operatorname{tr}\left(X_{n-1}|\Lambda|^{-2}\right)+\left(\gamma_{2}-\gamma_{3}\right) N \operatorname{tr} \log X_{2}-\gamma_{2} N \operatorname{tr} \log X_{3}-\gamma_{3} N \operatorname{tr} \log \left(X_{4} \cdots X_{n-1}\right)\right\}
\end{aligned}
$$

We use this expression when deriving the spectral curve equation in the next section.

2.2. The braid-group action. We now address the following natural question: the orders of the ramification points are not fixed a priori. So, we must be able to permute matrices in the above matrix chain. This makes our matrix chain interaction different from the standard matrix chain interaction which is of the form $\operatorname{tr} M_{i} M_{i+1}$ and which lacks such a symmetry. Indeed, we have a transformation corresponding to an elementary permutation of (neighbour) branching points depicted in Fig. 3. For the matrix chain, this transformation is described by a braid-group generator:

$$
\beta_{i}:\left\{X_{i} \rightarrow X_{i-1} X_{i}^{-1} X_{i+1} ; X_{j} \rightarrow X_{j}, j \neq i\right\} .
$$

It is easy to see that the action of each such generator with $3 \geq i \geq n-2$ leaves the action (2.6) invariant.

2.3. The eigenvalue representation and the KP hierarchy. Now we proceed further expressing integral (2.6) in terms of eigenvalues $x_{i}^{(k)}$ of the matrices $X_{k}, k=2, \ldots, n-1$.

We apply the Mehta-Itzykson-Zuber integration formula to every term in the chain of matrices in (2.6). Taking into account that, for instance, the integral over the unitary group for the term $e^{-N \operatorname{tr} X_{k} X_{k+1}^{-1}}$ gives

$$
\int D U e^{-N \sum_{i, j=1}^{\gamma_{3} N} U_{i j} x_{i}^{(k)} U_{i j}^{*}\left[x_{j}^{(k+1)}\right]^{-1}}=\frac{\operatorname{det}_{i, j}\left[e^{-N x_{i}^{(k)} / x_{j}^{(k+1)}}\right]}{\Delta\left(x^{(k)}\right) \Delta\left(1 / x^{(k+1)}\right)}
$$

and that $1 / \Delta\left(1 / x^{(k+1)}\right)=\prod_{i=1}^{\gamma_{3} N}\left[x_{i}^{(k+1)}\right]^{\gamma_{3} N-1} / \Delta\left(x^{(k+1)}\right)$ we eventually write the expression in terms of eigenvalues of the matrices $X_{k}$ :

$$
\int_{0}^{\infty} \prod_{i=1}^{\gamma_{3} N} d x_{i}^{(2)} \frac{\Delta\left(x^{(2)}\right)}{\Delta\left(|\Lambda|^{-2}\right)} \prod_{k=3}^{n-1}\left(\prod_{i=1}^{\gamma_{3} N} \frac{d x_{i}^{(k)}}{x_{i}^{(k)}}\right) \times
$$




$$
\times \prod_{i=1}^{\gamma_{3} N}\left[\left(x_{i}^{(2)} / x_{i}^{(3)}\right)^{\left(\gamma_{2}-\gamma_{3}\right) N} e^{N \sum_{r=1}^{\infty} \frac{t_{r}}{r}\left(x_{i}^{(2)}\right)^{r}-N x_{i}^{(2)} / x_{i}^{(3)}-\cdots-N x_{i}^{(n-2)} / x_{i}^{(n-1)}-N x_{i}^{(n-1)}|\Lambda|_{i}^{-2}}\right]
$$

Finally, if we introduce logarithmic quantities

$$
\varphi_{i}^{(r)}=\log x_{i}^{(r)}, \quad r=3, \ldots, n-1,
$$

we can rewrite integral (2.8) in a more transparent form resembling that of the Toda chain Lagrangian:

$$
\begin{aligned}
& \int_{0}^{\infty} \prod_{i=1}^{\gamma_{3} N} d x_{i}^{(2)} \frac{\Delta\left(x^{(2)}\right)}{\Delta\left(|\Lambda|^{-2}\right)} \prod_{i=1}^{\gamma_{3} N}\left[\int_{-\infty}^{\infty} \prod_{k=3}^{n-1} d \varphi_{i}^{(k)} \times\right. \\
& \quad \times \exp \left[N \sum_{r=1}^{\infty} \frac{t_{r}}{r}\left(x_{i}^{(2)}\right)^{r}+\left(\gamma_{2}-\gamma_{3}\right) N \log x_{i}^{(2)}-\left(\gamma_{2}-\gamma_{3}\right) N \varphi_{i}^{(3)}\right. \\
&\left.\left.\quad-N x_{i}^{(2)} e^{-\varphi_{i}^{(3)}}-N e^{\varphi_{i}^{(3)}-\varphi_{i}^{(4)}}-\cdots-N e^{\varphi_{i}^{(n-2)}-\varphi_{i}^{(n-1)}}-N e^{\varphi_{i}^{(n-1)}}|\Lambda|_{i}^{-2}\right]\right] .
\end{aligned}
$$

In this form it is clear that all integrals w.r.t. $\varphi_{i}^{(k)}$ are convergent. Performing intermediate integrations w.r.t. variables $\varphi_{i}^{(k)}$ for fixed $i$ and $k$ from 3 to $n-1$ we obtain that every monomial in the Vandermonde determinant in the numerator will be replaced by the corresponding function of the Meijer G-function type, as in [2].

\section{SpeCtral CURVE AND topological RECURsion}

In this section, we propose a method for deriving the spectral curve of model (2.6), adapting the technique of [23] to our case of a nonstandard interaction between matrices in the matrix chain. In this paper, we restrict ourselves to a technically more transparent case of the threematrix model given by the integral

$$
\int D M_{1} D M_{2} D M_{3} D M_{4} e^{N \operatorname{tr}\left[V\left(M_{1}\right)+M_{1} M_{2}^{-1}-\gamma_{2} \log M_{2}+M_{2} M_{3}^{-1}+M_{3} M_{4}^{-1}+U\left(M_{4}\right)\right]},
$$

where the integrations are performed w.r.t. positive-definite Hermitian matrices of size $\gamma_{3} N \times$ $\gamma_{3} N$ and potentials $V(x)$ and $U(x)$ are two Laurent polynomials of the respective positive degrees $n$ and $r$ (this consideration can be easily generalised to the case where $V^{\prime}(x)$ and $U^{\prime}(x)$ are two rational functions).

The model (3.1) satisfies (see [28], [26]) equations of the two-dimensional Toda chain hierarchy, and in fact these two classes of models are closely related, so solving the problem of finding the spectral curve in one model can be translated in a standard way to solving the corresponding problem in the other model. Because finding spectral curves for multi-matrix models is more transparent technically than finding spectral curves for models with external matrix fields, we stay with the first choice.

3.1. Field variations and loop equations. We consider the following variations of the matrix fields $M_{i}$ :

$$
\begin{aligned}
\delta M_{1} & =\frac{1}{x-M_{1}} \xi\left(\left[\widehat{M}_{1}\right]\right), \\
\delta M_{2} & =M_{2} \frac{1}{x-M_{1}} \eta\left(\left[\widehat{M}_{2}\right]\right), \\
\delta M_{3} & =M_{3} \frac{1}{x-M_{1}} \rho\left(\left[\widehat{M}_{3}\right]\right),
\end{aligned}
$$




$$
\delta M_{4}=\frac{1}{x-M_{1}} \chi\left(\left[\widehat{M}_{4}\right]\right)
$$

where we take $\xi, \eta, \rho$, and $\chi$ to be Laurent polynomials in all but one of arguments $M_{i}$. We indicate the omitted argument by the symbol $\left[\widehat{M}_{i}\right]$. For example, a function $\eta\left(\left[\widehat{M}_{2}\right]\right)$ is a Laurent polynomial in $M_{1}, M_{3}$, and $M_{4}$. We introduce the standard notation for the leading term of the $1 / N^{2}$-expansion of the one-loop mean of the matrix field $M_{1}$ :

$$
\omega_{1}(x):=\frac{1}{N}\left\langle\operatorname{tr} \frac{1}{x-M_{1}}\right\rangle_{0} .
$$

Here and hereafter, the subscript 0 of a correlation function indicates the contribution of the leading order of the $1 / N^{2}$-expansion. A single trace symbol in the brackets pertains to the whole expression inside the corresponding brackets.

The exact loop equations obtained upon variations (3.2) read

$$
\begin{aligned}
& \frac{1}{N^{2}}\left\langle\operatorname{tr} \frac{1}{x-M_{1}} \operatorname{tr} \frac{1}{x-M_{1}} \xi\left(\left[\widehat{M}_{1}\right]\right)\right\rangle+\left[\omega_{1}(x)+V^{\prime}(x)\right]\left\langle\operatorname{tr} \frac{1}{x-M_{1}} \xi\left(\left[\widehat{M}_{1}\right]\right)\right\rangle \\
& \quad+\left\langle\operatorname{tr} \frac{V^{\prime}\left(M_{1}\right)-V^{\prime}(x)}{x-M_{1}} \xi\left(\left[\widehat{M}_{1}\right]\right)\right\rangle+\left\langle\operatorname{tr} M_{2}^{-1} \frac{1}{x-M_{1}} \xi\left(\left[\widehat{M}_{1}\right]\right)\right\rangle=0 ; \\
& \left\langle\operatorname{tr} \frac{-1}{x-M_{1}} \eta\left(\left[\widehat{M}_{2}\right]\right) M_{2}^{-1} M_{1}\right\rangle+\left\langle\operatorname{tr} M_{3}^{-1} M_{2} \frac{1}{x-M_{1}} \eta\left(\left[\widehat{M}_{2}\right]\right)\right\rangle \\
& \quad+\left(\gamma_{2}-\gamma_{3}\right)\left\langle\operatorname{tr} \frac{1}{x-M_{1}} \eta\left(\left[\widehat{M}_{2}\right]\right)\right\rangle=0 ; \\
& \left\langle\operatorname{tr} \frac{1}{x-M_{1}} \rho\left(\left[\widehat{M}_{3}\right]\right) M_{3}^{-1} M_{2}\right\rangle=\left\langle\operatorname{tr} \frac{1}{x-M_{1}} \rho\left(\left[\widehat{M}_{3}\right]\right) M_{4} M_{3}\right\rangle ; \\
& \left\langle\operatorname{tr} M_{3} \frac{1}{x-M_{1}} \chi\left(\left[\widehat{M}_{4}\right]\right)\right\rangle+\left\langle\operatorname{tr} U^{\prime}\left(M_{4}\right) \frac{1}{x-M_{1}} \chi\left(\left[\widehat{M}_{4}\right]\right)\right\rangle=0 .
\end{aligned}
$$

A complete information on the model is encoded in these loop equations; solving them we can develop the topological recursion procedure for evaluating all terms of the $1 / N^{2}$-expansion. Amazingly enough, the whole topological expansion can be constructed out of the information contained in the leading-order term (the planar approximation, or $g=0$ ). So, as soon as we derive the spectral curve endowed with two differentials $d x$ and $\omega_{1}(x) d x$, we can construct a complete genus expansion in $N^{2-2 g}$ to any desired genus $g$ using the topological recursion method of [22], 14], 15] (see also [4]; the novel abstract algebraic approach to this method was developed in [10]). So, finding the spectral curve in terms of $x$ and $\omega_{1}(x)$ is a definitive step.

Because we obtain the spectral curve in the large- $N$ limit, we disregard the first term in (3.4), which is of the next order in $1 / N^{2}$. All other terms in all three equations contribute to the leading order.

3.2. Finding the spectral curve. Calculations are rather tedious and consist in substitutions of various functions $\xi, \eta, \rho$, and $\chi$ into the above loop equations. In order to shorten the writing and for the future convenience, we introduce the following notation:

$$
\begin{aligned}
\mathbf{a} & :=\left\langle\operatorname{tr} \frac{1}{x-M_{1}} \frac{U^{\prime}\left(M_{4}\right)-U^{\prime}(z)}{M_{4}-z}\right\rangle_{0}, \\
\mathbf{b} & :=\left\langle\operatorname{tr} M_{2}^{-1} \frac{1}{x-M_{1}} \frac{U^{\prime}\left(M_{4}\right)-U^{\prime}(z)}{M_{4}-z}\right\rangle_{0},
\end{aligned}
$$




$$
\begin{aligned}
& \mathbf{c}:=\left\langle\operatorname{tr} M_{3} \frac{1}{x-M_{1}} \frac{U^{\prime}\left(M_{4}\right)-U^{\prime}(z)}{M_{4}-z}\right\rangle_{0}, \\
& \mathbf{d}:=\left\langle\operatorname{tr} M_{2} \frac{1}{x-M_{1}} \frac{U^{\prime}\left(M_{4}\right)-U^{\prime}(z)}{M_{4}-z}\right\rangle_{0} .
\end{aligned}
$$

We next perform several substitutions enabling us to produce the required identities; in all identities below we keep only leading terms in the large- $N$ limit.

(i) The first substitution (in (3.4)) is

$$
\xi\left(\left[\widehat{M}_{1}\right]\right)=\frac{U^{\prime}\left(M_{4}\right)-U^{\prime}(z)}{M_{4}-z}: \quad\left[\omega_{1}(x)+V^{\prime}(x)\right] \mathbf{a}+P_{n, m}(x, z)+\mathbf{b}=0,
$$

where

$$
P_{n, m}(x, z):=\left\langle\operatorname{tr} \frac{U^{\prime}\left(M_{4}\right)-U^{\prime}(z)}{M_{4}-z} \frac{V^{\prime}\left(M_{1}\right)-V^{\prime}(x)}{x-M_{1}}\right\rangle_{0}
$$

is a Laurent polynomial in $x$ and $z$ of degrees $n$ and $m$ that are smaller by two than the corresponding degrees of the potentials $V(x)$ and $U(z)$.

(ii) The second substitution (in (3.5)) is

$$
\begin{aligned}
& \eta\left(\left[\widehat{M}_{2}\right]\right)=\frac{U^{\prime}\left(M_{4}\right)-U^{\prime}(z)}{M_{4}-z}: \\
& \left\langle\operatorname{tr} \frac{-M_{1}}{x-M_{1}} \frac{U^{\prime}\left(M_{4}\right)-U^{\prime}(z)}{M_{4}-z} M_{2}^{-1}\right\rangle_{0}+\left\langle\operatorname{tr} M_{3}^{-1} M_{2} \frac{1}{x-M_{1}} \frac{U^{\prime}\left(M_{4}\right)-U^{\prime}(z)}{M_{4}-z}\right\rangle_{0}+\left(\gamma_{2}-\gamma_{3}\right) \mathbf{a}=0 .
\end{aligned}
$$

(iii) The third substitution (in 3.6) is

$$
\begin{aligned}
& \rho\left(\left[\widehat{M}_{3}\right]\right)=\frac{U^{\prime}\left(M_{4}\right)-U^{\prime}(z)}{M_{4}-z}: \\
& \left\langle\operatorname{tr} M_{3}^{-1} M_{2} \frac{1}{x-M_{1}} \frac{U^{\prime}\left(M_{4}\right)-U^{\prime}(z)}{M_{4}-z}\right\rangle_{0}=\left\langle\operatorname{tr} \frac{1}{x-M_{1}} \frac{U^{\prime}\left(M_{4}\right)-U^{\prime}(z)}{M_{4}-z} M_{4} M_{3}\right\rangle_{0} .
\end{aligned}
$$

Combining (ii) and (iii), we obtain

$$
\widehat{Q}_{m}(z)-x \mathbf{b}+\left(\gamma_{2}-\gamma_{3}\right) \mathbf{a}+\left\langle\operatorname{tr} \frac{1}{x-M_{1}} \frac{U^{\prime}\left(M_{4}\right)-U^{\prime}(z)}{M_{4}-z}\left(M_{4}-z\right) M_{3}\right\rangle_{0}+z \mathbf{c}=0
$$

where we have introduced the polynomial

$$
\widehat{Q}_{m}(z):=\left\langle\operatorname{tr} \frac{U^{\prime}\left(M_{4}\right)-U^{\prime}(z)}{M_{4}-z} M_{2}^{-1}\right\rangle_{0}
$$

Using the loop equation (3.7), we can replace $U^{\prime}\left(M_{4}\right)$ in any expression not containing other insertions of the matrix $M_{4}$ by $-M_{3}$, so (3.14) takes the form

$$
-x \mathbf{b}+\left(\gamma_{2}-\gamma_{3}\right) \mathbf{a}+z \mathbf{c}+\widehat{Q}_{m}(z)-\left\langle\operatorname{tr} \frac{1}{x-M_{1}}\left[M_{3}^{2}+M_{3} U^{\prime}(z)\right]\right\rangle_{0}=0
$$

(iv) The fourth substitution (in 3.4) is

$$
\begin{aligned}
& \xi\left(\left[\widehat{M}_{1}\right]\right)=\frac{U^{\prime}\left(M_{4}\right)-U^{\prime}(z)}{M_{4}-z} M_{3}: \\
& {\left[\omega_{1}(x)+V^{\prime}(x)\right] \mathbf{c}+\widehat{P}_{n, m}(x, z)+\mathbf{b}+\left\langle\operatorname{tr} M_{3} M_{2}^{-1} \frac{1}{x-M_{1}} \frac{U^{\prime}\left(M_{4}\right)-U^{\prime}(z)}{M_{4}-z}\right\rangle_{0}=0}
\end{aligned}
$$


where

$$
\widehat{P}_{n, m}(x, z):=\left\langle\operatorname{tr} \frac{U^{\prime}\left(M_{4}\right)-U^{\prime}(z)}{M_{4}-z} \frac{V^{\prime}\left(M_{1}\right)-V^{\prime}(x)}{x-M_{1}} M_{3}\right\rangle_{0} .
$$

(v) The fifth substitution (in (3.5)) is

$$
\begin{aligned}
& \eta\left(\left[\widehat{M}_{2}\right]\right)=\frac{U^{\prime}\left(M_{4}\right)-U^{\prime}(z)}{M_{4}-z} M_{3}: \\
& \left\langle\operatorname{tr} \frac{-M_{1}}{x-M_{1}} \frac{U^{\prime}\left(M_{4}\right)-U^{\prime}(z)}{M_{4}-z} M_{3} M_{2}^{-1}\right\rangle_{0}+\mathbf{d}+\left(\gamma_{2}-\gamma_{3}\right) \mathbf{c}=0 .
\end{aligned}
$$

Introducing the polynomial in $z$

$$
\widehat{\widehat{Q}}_{m}(z):=\left\langle\operatorname{tr} \frac{U^{\prime}\left(M_{4}\right)-U^{\prime}(z)}{M_{4}-z} M_{3} M_{2}^{-1}\right\rangle_{0}
$$

we can rewrite 3.19$)$ in the form

$$
\widehat{\widehat{Q}}_{m}(z)-x\left\langle\operatorname{tr} \frac{1}{x-M_{1}} \frac{U^{\prime}\left(M_{4}\right)-U^{\prime}(z)}{M_{4}-z} M_{3} M_{2}^{-1}\right\rangle_{0}+\mathbf{d}+\left(\gamma_{2}-\gamma_{3}\right) \mathbf{c}=0
$$

and expressing the term in angular brackets using (3.17), we come to the equation

$$
x\left[\omega_{1}(x)+V^{\prime}(x)\right] \mathbf{c}+\left(\gamma_{2}-\gamma_{3}\right) \mathbf{c}+\mathbf{d}+x \widehat{P}_{n, m}(x, z)+\widehat{\widehat{Q}}_{m}(z)=0 .
$$

The sixth and the last substitution (in (3.4) ) is

$$
\xi\left(\left[\widehat{M}_{1}\right]\right)=\frac{U^{\prime}\left(M_{4}\right)-U^{\prime}(z)}{M_{4}-z} M_{2}: \quad\left[\omega_{1}(x)+V^{\prime}(x)\right] \mathbf{d}+\widehat{\widehat{P}}_{n, m}(x, z)+\mathbf{a}=0,
$$

where

$$
\widehat{\widehat{P}}_{n, m}(x, z):=\left\langle\operatorname{tr} \frac{U^{\prime}\left(M_{4}\right)-U^{\prime}(z)}{M_{4}-z} \frac{V^{\prime}\left(M_{1}\right)-V^{\prime}(x)}{x-M_{1}} M_{2}\right\rangle_{0} .
$$

We now express b from (3.10) ending up with the system of three equations (3.16), (3.21), and (3.22) on three variables $\mathbf{a}, \mathbf{c}$, and $\mathbf{d}$ :

$$
\begin{aligned}
& {\left[x\left[\omega_{1}(x)+V^{\prime}(x)\right]+\left(\gamma_{2}-\gamma_{3}\right)\right] \mathbf{a}+z \mathbf{c}} \\
& =-x P_{n, m}(x, z)-\widehat{Q}_{m}(z)+\left\langle\operatorname{tr} \frac{1}{x-M_{1}}\left[M_{3}^{2}+M_{3} U^{\prime}(z)\right]\right\rangle_{0} \\
& {\left[x\left[\omega_{1}(x)+V^{\prime}(x)\right]+\left(\gamma_{2}-\gamma_{3}\right)\right] \mathbf{c}+\mathbf{d}=-x \widehat{P}_{n, m}(x, z)-\widehat{\widehat{Q}}_{m}(z)} \\
& \mathbf{a}+\left[\omega_{1}(x)+V^{\prime}(x)\right] \mathbf{d}=-\widehat{\widehat{P}}_{n, m}(x, z) .
\end{aligned}
$$

We now treat the system of equations (3.24) as a system of three linear equations on three unknowns $\mathbf{a}, \mathbf{c}$, and $\mathbf{d}$. We still have a free parameter $z$ and the idea is to choose this parameter in the way to make the corresponding system degenerate. Then, the condition of solvability w.r.t. the right-hand sides of system (3.24) produces the equation of the spectral curve.

Introducing the shorthand notation

$$
r(x):=x\left[\omega_{1}(x)+V^{\prime}(x)\right]+\left(\gamma_{2}-\gamma_{3}\right), \quad s(x):=\omega_{1}(x)+V^{\prime}(x)
$$

the system determinant is

$$
\left|\begin{array}{ccc}
r(x) & 0 & z \\
0 & 1 & r(x) \\
1 & s(x) & 0
\end{array}\right|=-z-r^{2}(x) s(x)
$$


which immediately gives

$$
z=-r^{2}(x) s(x),
$$

and the condition of solvability of the degenerate system (3.24), or, equivalently, the equation of the spectral curve is

$$
\begin{aligned}
-x P_{n, m}(x, z)-\widehat{Q}_{m}(z)+\left\langle\operatorname{tr} \frac{1}{x-M_{1}}\left[M_{3}^{2}+M_{3} U^{\prime}(z)\right]\right\rangle_{0} \\
+r(x) \widehat{\widehat{P}}_{n, m}(x, z)-s(x) r(x)\left[x \widehat{P}_{n, m}(x, z)+\widehat{\widehat{Q}}_{m}(z)\right]=0,
\end{aligned}
$$

where $P_{n, m}(x, z), \widehat{P}_{n, m}(x, z), \widehat{\widehat{P}}_{n, m}(x, z), \widehat{Q}_{m}(z)$, and $\widehat{\widehat{Q}}_{m}(z)$ are the polynomials given by the corresponding formulas (3.11), (3.18), (3.23), (3.15), and (3.20), $r(x)$ and $s(x)$ are defined in (3.25), and $z=-r^{2}(x) s(x)$ (3.26). In order to complete the construction, we need only to evaluate the quantities $\left\langle\operatorname{tr} \frac{1}{x-M_{1}} M_{3}^{k}\right\rangle_{0}$ for $k=1,2$. The method for finding quantities $\left\langle\operatorname{tr} \frac{1}{x-M_{1}} M_{2}^{k}\right\rangle_{0}$ for any integer $k$ using the loop equation (3.4) alone was presented in [8]. In the next subsection, we develop this method further and show that we can evaluate similar quantities with insertions of the third, not second, matrix $M_{3}$ using only two the first loop equations (3.4) and (3.5).

3.3. Finding $\left\langle\operatorname{tr} \frac{1}{x-M_{1}} M_{3}^{k}\right\rangle_{0}$. We Introduce the convenient notation

$$
f_{k, r}(x):=\left\langle\operatorname{tr} \frac{1}{x-M_{1}} M_{3}^{k} M_{2}^{r}\right\rangle_{0}, \quad f_{0,0}(x)=\omega_{1}(x),
$$

and the polynomials and constants

$$
R_{k, r}(x):=\left\langle\operatorname{tr} \frac{V^{\prime}(x)-V^{\prime}\left(M_{1}\right)}{x-M_{1}} M_{3}^{k} M_{2}^{r}\right\rangle_{0}, \quad C_{k}:=\left\langle\operatorname{tr} M_{3}^{k} M_{2}^{-1}\right\rangle_{0} .
$$

Performing the substitution $\xi\left(\left[\widehat{M}_{1}\right]\right)=M_{3}^{k} M_{2}^{r}$ into (3.4), we obtain:

$$
\left[\omega_{1}(x)+V^{\prime}(x)\right] f_{k^{\prime}, r^{\prime}}(x)+R_{k^{\prime}, r^{\prime}}(x)+f_{k^{\prime}, r^{\prime}-1}(x)=0, \quad k^{\prime}, r^{\prime} \in \mathbb{Z} .
$$

From the relation (3.29) we can evaluate (see [8]) $f_{0, r}(x)$ for any $r \in \mathbb{Z}$ using that $f_{0,0}(x)=\omega_{1}(x)$.

We next consider the substitution $\eta\left(\left[\widehat{M}_{2}\right]\right)=M_{3}^{k}$ in (3.5). It gives

$$
\left\langle\operatorname{tr} \frac{-M_{1}}{x-M_{1}} M_{3}^{k} M_{2}^{-1}\right\rangle_{0}+\left\langle\operatorname{tr} \frac{-M_{1}}{x-M_{1}} M_{3}^{k-1} M_{2}\right\rangle_{0}+\left(\gamma_{2}-\gamma_{3}\right)\left\langle\operatorname{tr} \frac{-M_{1}}{x-M_{1}} M_{3}^{k}\right\rangle_{0}=0,
$$

and using the same trick of adding and subtracting $x$ to the nominator of the fraction in the first term, we obtain the second recurrent equation

$$
C_{k}-x f_{k,-1}(x)+f_{k-1,1}(x)+\left(\gamma_{2}-\gamma_{3}\right) f_{k, 0}(x)=0 .
$$

Using (3.29) and (3.30) we can find $f_{k, 0}(x), f_{k, 1(x)}$, and $f_{k,-1}(x)$ for all integer $k$. For our purposes here we need only $f_{k, 0}(x)$. Let us fix $k \in \mathbb{Z}$. We use (3.29) with $\left(k^{\prime}, r^{\prime}\right)=(k-1,1)$ to express $f_{k-1,1}(x)$ through $f_{k-1,0}(x)$ and (3.29) with $\left(k^{\prime}, r^{\prime}\right)=(k, 0)$ to express $f_{k,-1}(x)$ through $f_{k, 0}(x)$. The resulting recursion relation takes the form

$$
\begin{aligned}
{\left[x \left[\omega_{1}(x)\right.\right.} & \left.\left.+V^{\prime}(x)\right]+\left(\gamma_{2}-\gamma_{3}\right)\right]\left[\omega_{1}(x)+V^{\prime}(x)\right] f_{k, 0}(x) \\
& =f_{k-1,0}(x)+R_{k-1,1}(x)+\left[\omega_{1}(x)+V^{\prime}(x)\right]\left(x R_{k, 0}(x)+C_{k}\right)
\end{aligned}
$$

Again, suppling it with the initial condition $f_{0,0}(x)=\omega_{1}(x)$ we express all $f_{k, 0}(x)$ in terms of $r(x)$ and $s(x)$ (3.25) and polynomials $R_{k^{\prime}, 0}(x), R_{k^{\prime}, 1}(x)$ and constants $C_{k^{\prime}}$ (3.28). 


\section{COnClusion}

We developed further the construction of a matrix chain representation of the generating functions for hypergeometric Hurwitz numbers started in [8]. Our calculations for the case of $n=5$ distinct branching points demonstrate how we can evaluate the corresponding spectral curve in the case of any fixed $n$; all these curves are going to be rational.

There could be several directions of development of this technique. First, and most important one, is that the method proposed in this paper is still far from being effective: we obtain a rational spectral curve with, albeit finite, but large number of "free" parameters. We know that we can actually fix all these parameters if we assume the spectral curve to be of genus zero (which is a natural assumption for theories that are perturbative excitations of a Gaussian free-field theory) and if we take into account the asymptotic expansion of the $y d x$ differential at the infinity, which is completely governed by the potential $V^{\prime}(x)$.

Another direction of development is related to possible applications of generating functions of type (1.2) in geometry. It is known that in the case of so-called clean Belyi morphisms, these functions are related [7] to the free energy of the Kontsevich-Penner matrix model [16], [17, which is known $([13],[36$, ,21] ) to be the generating function of the numbers of integer points in moduli spaces $\mathcal{M}_{g, s}$ of curves of genus $g$ with $s$ holes with fixed (integer) perimeters. It is tempting to explore possible relations of these discretization patterns to cut-and-join operators of [42] and [6] in the case of hypergeometric Hurwitz numbers and to Hodge integrals of [29]. We can also try to explore a possibility to apply matrix model methods of our series of papers to studying generalizations of Hurwitz numbers, including weighted Hurwitz numbers [3] and their multispecies generalizations [27].

\section{ACKNOWLEDGMENTS}

The authors acknowledge support from the ERC Advance Grant 291092 "Exploring the Quantum Universe" (EQU) as well as support of FNU, the Free Danish Research Council, from the grant "Quantum Geometry". The work of L.Ch. was supported in part by the Russian Foundation for Basic Research (Grant No. 18-01-00273a).

\section{REFERENCES}

[1] G. Akemann, J. Ipsen, and M. Kielburg, Products of rectangular random matrices: Singular values and progressive scattering, Phys. Rev. E 88 (2013) 052118; arXiv:1307.7560.

[2] G. Akemann, M. Kielburg, and L. Wej, Singular value correlation functions for products of Wishart matrices, J. Phys. A: Math. Theor. 46 (2013) 275205; arXiv:1303.5694.

[3] A. Alexandrov, G. Chapuy, B. Eynard, and J. Harnad, Fermionic approach to weighted Hurwitz numbers and topological recursion, arXiv:1706.00958, 57pp.

[4] A. Alexandrov, A. Mironov and A. Morozov, Partition functions of matrix models as the first special functions of String Theory I. Finite size Hermitean 1-matrix model, Int. J. Mod. Phys. A19 (2004) 4127-4165.

[5] A. Alexandrov, A. Mironov, A. Morozov, and S. Natanzon, Integrability of Hurwitz partition functions. I. Summary, J. Phys. A: Math. Theor. 45 (2012) 045209; arXiv:1103.4100.

[6] A. Alexandrov, A. Mironov, A. Morozov, and S. Natanzon, On KP-integrable Hurwitz functions, JHEP 11 (2014) 080; arXiv:1405.1395.

[7] J. Ambjørn and L. Chekhov, The matrix model for dessins d'enfants, Ann. Inst. Henri Poincaré, Comb. Phys. Interact. 1 (2014) 337-361; DOI 10.4171/AIHPD/10.

[8] J. Ambjørn and L. Chekhov, The matrix model for hypergeometric Hurwitz numbers, Theor. Math. Phys. 181(3) (2014) 1486-1498.

[9] J. Ambjørn, C. F. Kristjansen, and Y. M. Makeenko, Higher genus correlators for the complex matrix model, Mod. Phys. Lett. A7 (1992) 3187-3203; hep-th/9207020.

[10] J. E. Andersen, G. Borot, L. Chekhov, and N. Orantin, The ABCD of topological recursion, arXiv:1703.03307, 75pp.

[11] G. Belyi, On Galois extension of a maximal cyclotomic field, USSR Math. Izvestiya 14:2 (1980) 247-256. 
[12] L.Chekhov, Matrix models with hard walls: Geometry and solutions, J. Phys. A 39 (2006) 8857-8894; hep-th/0602013

[13] L. Chekhov, Matrix models tools and geometry of moduli spaces, Acta Appl. Mathematicae 48 (1997) 33-90; hep-th/9509001.

[14] L. Chekhov, B. Eynard, Hermitean matrix model free energy: Feynman graph technique for all genera, JHEP 0603:014 (2006); hep-th/0504116.

[15] L. Chekhov, B. Eynard, and N. Orantin, Free energy topological expansion for the 2-matrix model, JHEP 12(2006)053; hep-th/0603003.

[16] L. Chekhov and Yu. Makeenko, The multicritical Kontsevich-Penner model, Mod. Phys. Lett. A7 (1992) 1223-1236; hep-th/9201033.

[17] L. Chekhov and Yu. Makeenko, A hint on the external field problem for matrix models, Phys. Lett. B278 (1992) 271-278; hep-th/9202006

[18] P. Di Francesco and C. Itzykson, A generating function for fatgraphs, Ann. Inst. H. Poincaré, Phys. Teor. 59 (1993) 117-140.

[19] R. De Mello Koch and S. Ramgoolam, From Matrix Models and quantum fields to Hurwitz space and the absolute Galois group, arXiv:1002.1634

[20] R. De Mello Koch, S. Ramgoolam, and C. Wen, On the refined counting of graphs on surfaces, Nucl. Phys. B 870(3) (2013) 530-581; arXiv:1209.0334.

[21] Norman Do and Paul Norbury, Pruned Hurwitz numbers, Trans. AMS, 370(5) (2018) 3053-3084; arXiv:1312.7561.

[22] B. Eynard, All genus correlation functions for the hermitian 1-matrix model, JHEP 0411:031 (2004).

[23] B. Eynard and A. Prats Ferrer, Topological expansion of the chain of matrices, JHEP 0907 (2009) 096; arXiv:0805.1368 2 .

[24] I. P. Goulden and D. M. Jackson, The KP hierarchy, branched covers, and triangulations, arXiv:0803.3980

[25] A. Grothendieck, Esquisse d'un programme, Geometric Galois Action, Cambridge Univ. Press, Cambridge (1997) 5-48.

[26] M. Guay-Paquet and J. Harnad, Generating functions for weighted Hurwitz numbers, J. Math. Phys. 58 (2017) 083503; arXiv:1408.6766

[27] J. Harnad, Multispecies weighted Hurwitz numbers, SIGMA, 11 (2015) 097, 19pp; arXiv:1504.07512.

[28] J. Harnad and A. Yu. Orlov, Hypergeometric $\tau$-functions, Hurwitz numbers and enumeration of paths, Commun. Math. Phys. 338 (2015) 267-284; arXiv:1407.7800

[29] M. Kazarian, KP hierarchy for Hodge integrals, Adv. Math. 221 (2009) 1-21.

[30] M. Kazarian and P. Zograf, Virasoro constraints and topological recursion for Grothendieck's dessin counting, Lett. Math. Phys. 105 (2015) 1057-1084; arXiv:1406.5976.

[31] S. Kharchev, A.Marshakov, A.Mironov, and A.Morozov, Generalized Kazakov-Migdal-Kontsevich model: Group theory aspects, Int. J. Mod. Phys. A10 (1995) 2015-2045; hep-th/9312210.

[32] S. Kharchev, A.Marshakov, A.Mironov, A.Morozov, and A. Zabrodin, Unification of all string models with $c<1$, Phys.Lett., 275B (1992) 311-314.

[33] S. Kharchev, A.Marshakov, A.Mironov, A.Morozov, and A. Zabrodin, Towards unified theory of 2d gravity, Nucl. Phys., B380 (1992) 181-240.

[34] V. A. Marchenko and L. A. Pastur, Distribution of eigenvalues for some sets of random matrices, Math. USSR-Sb. 1 (1967) 457-483.

[35] A. Mironov, A. Morozov, and G. Semenoff, Unitary matrix integrals in the framework of Generalized Kontsevich Model. I. Brezín-Gross-Witten model, Int. J. Mod. Phys. A10 (1995) 2015-2040.

[36] P. Norbury, Counting lattice points in the moduli space of curves, Math. Res. Lett. 17 (2010) 467-481.

[37] A. Okounkov, Toda equations for Hurwitz numbers, Math. Res. Lett. 7 (2000) 447-453.

[38] A. Okounkov and R. Pandharipande, Gromov-Witten theory, Hurwitz numbers, and completed cycles, Ann. Math. 163 (2006) 517-590; math.AG/0204305.

[39] A. Orlov and D. M. Shcherbin, Hypergeometric solutions of soliton equations, Theor. Math. Phys. 128 (2001) 906-926.

[40] A. Orlov, Hypergeometric functions as infinite-soliton tau functions, Theor. Math. Phys. 146 (2006) 183-206.

[41] K. Takasaki, Initial value problem for the Toda lattice hierarchy, Adv. Studies Pure Math. 4 (1984) 139-163.

[42] P. G. Zograf, Enumeration of Grothendieck's dessins and KP hierarchy, Intl. Math. Res. Notices 24 (2015) 13533-13544; arXiv:1312:2538v2. 